\title{
Investigation of Spherical and Cylindrical Luneburg Lens Antennas by Green's Function Method
}

\author{
Sergey Knyazev, Alexey Korotkov, Boris Panchenko, Sergey Shabunin \\ Institute of Radioelectronics and Information Technologies \\ Ural Federal University \\ Ekaterinburg, Russia \\ shab@ieee.org
}

\begin{abstract}
Luneburg lens antenna radiation fields are calculated with Green's functions of spherical and cylindrical layered structures. Electric field components for Luneburg lenses excited by a linear and circular polarized antenna are analyzed. Copolarized and cross-polarized field radiation patterns are shown. Reflection from the lens, losses in the lens material, spillover and polarization loss are taken into account for antenna gain calculation. The proposed method significantly reduces computing time for multilayered lens in comparison with the most commonly used in antenna design.
\end{abstract}

\section{INTRODUCTION}

Spherical and cylindrical Luneburg lenses are suitable for multiple beams and scanning high-gain antenna systems design. A refraction index of Luneburg lens varies as follows [1]

$$
n(r)=\sqrt{\varepsilon^{\prime}(r)}=\sqrt{2-(r / a)^{2}},
$$

where $\varepsilon^{\prime}$ is a relative dielectric constant of lens material at the point $r, r$ is a radial coordinate in a spherical or cylindrical coordinate system, $a$ is an outer lens radius in the corresponding coordinate system. Usually Luneburg lens is fabricated as a multilayer structure. The refractive index of the each layer is close enough to the law of Luneburg (1).

There are a lot of papers devoted to Luneburg lens antennas analysis with software as Ansoft HFSS [2], CST Microwave Studio [3] and so on. Authors make a note of rather much time processing. Fortunately, Luneburg lens may be well fitted by spherical or cylindrical coordinate system. It is rather simple to define radiation field by Green's functions method. Antenna radiation field is defined by integrating of electric and/or magnetic extraneous currents with Green's functions suitable to the spherical or cylindrical coordinate system. As extraneous currents electric or/and magnetic dipoles are used. For example crossed electric and magnetic dipoles form the Huygens source. This kind of the source is used for aperture antennas design as a horn antenna. A linear waveguide with slots for cylindrical Luneburg lens excitation is described by magnetic dipoles.

In this paper a universal algorithm with Green's functions of layered spherical and cylindrical structures is described for Luneburg lens antenna radiation problems solving. In comparison with Ansoft HFSS the offered method demands at least in one thousand times smaller time for calculations.
Radiation patterns, antenna gain, directivity, radiation efficiency and polarization properties may be rather quickly analyzed.

\section{SPECTRAL-DOMAIN FULL-WAVE APPROACH FOR}

\section{MULTILAYERED SPHERICAL AND CYLINDRICAL STRUCTURES}

Spectral-domain full-wave approach suggested in [4],[5] uses the rigorous decision of the Maxwell equations for radially non-uniform structures with arbitrary electrodynamic properties and any kind of electrical and magnetic current exciting. This method with matrix approach is suitable for computer calculations. It is easy to simulate layered structures, impedance surfaces, covers including metamaterials.

According to the suggested method the main electric and magnetic field component are chosen. Other components are defined with the main components. In the spherical coordinate system as the main radial electric $E_{r}$ and magnetic $H_{r}$ components are used. In cylindrical coordinates $E_{z}$ and $H_{z}$ are the main and the first one may be written as

$$
\begin{array}{r}
E_{z}(r, \varphi, z)=-\frac{1}{4 \pi^{2}} \sum_{m=-\infty}^{\infty} \int_{V^{\prime}} \int_{-\infty}^{\infty}\left[\Phi_{1}^{E}\left(m, h ; r, r^{\prime}\right)+\right. \\
\left.+\Phi_{2}^{E}\left(m, h ; r, r^{\prime}\right)\right] e^{-j m\left(\varphi-\varphi^{\prime}\right)} e^{-j h\left(z-z^{\prime}\right)} d h d v^{\prime}
\end{array}
$$

where functions $\Phi_{1}^{E}, \Phi_{2}^{E}$ contain information about sources, cylindrical structure dimensions and electrodynamic material properties. These functions may be considered as Green's functions. In (2) all primed symbols are source points. The integration is carried out with respect to source region $V^{\prime}$.

Electric field components for a spherical Luneburg lens antenna in the far zone are simplified by saddle-point method and become as follows

$$
\begin{gathered}
\mathbf{E}(\theta, \varphi, r)=E_{0} \cdot \frac{\mathrm{e}^{-i \cdot k_{\theta} r}}{\left(k_{0} r\right)} \sum_{n=1}^{\infty} \frac{2 n+1}{n(n+1)} \cdot \mathrm{e}^{i \frac{\pi}{2} n} \cdot \mathrm{e}^{i \varphi} . \\
\cdot\left\{\begin{array}{l}
{\left[\mathbf{a}_{\theta} \cdot \tau_{n}(\theta)+i \mathbf{a}_{\varphi} \cdot \pi_{n}(\theta)\right] \cdot\left(M_{n}^{\prime}+j M_{n}^{\prime \prime}\right)-} \\
{\left[\mathbf{a}_{\theta} \cdot \pi_{n}(\theta)+i \mathbf{a}_{\varphi} \cdot \tau_{n}(\theta)\right] \cdot\left(N_{n}^{\prime}+j N_{n}^{\prime}\right)}
\end{array}\right),
\end{gathered}
$$


where $k_{0}=2 \pi / \lambda$ is a wave number, $\mathbf{a}_{\theta}, \mathbf{a}_{\varphi}$ are unit vectors in the spherical coordinate system, $\tau_{n}(\theta)$ and $\pi_{n}(\theta)$ contain Legendre functions and derivatives of these function. Coefficients $M_{n}, N_{n}$ in (3) depend on the excitation type and layered structure properties.

All types of Luneburg lens exciters may be simulated at the corresponding choice of amplitudes and phases distribution of extraneous currents.

The model of equivalent electric and magnetic lines [5] for radially inhomogeneous magneto-dielectric structure modelling is used. Each layer is assigned with a part of radial line. Transmission matrices of equivalent lines and boundaries define electromagnetic waves propagation in each layer and between layers. The method makes unnecessary field calculation inside Luneburg lens. The saddle-point method is used for far field computation. All these essentially reduce computing time.

\section{NUMERICAL RESULTS}

Let us see polarization properties of the spherical Luneburg lens excited by circular polarized field from crossed dipoles with a reflector. The radiation patterns of co-polarized and cross-polarized field radiation of Luneburg lens are shown in Fig. 1 and Fig. 2 respectively. The lens has six layers. The outer radius is $k_{0} a=2 \pi$.

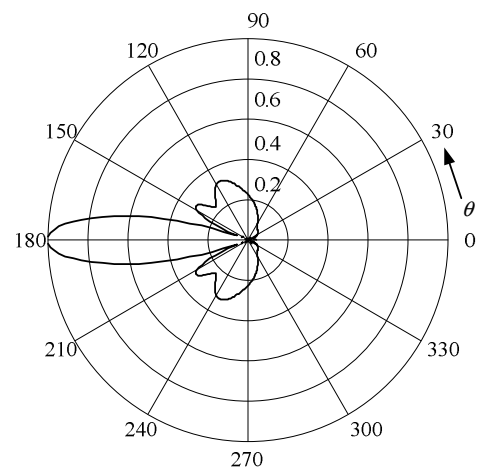

Fig. 1. Radiation pattern for main polarization of Luneburg lens exited by crossed dipoles with a reflector.

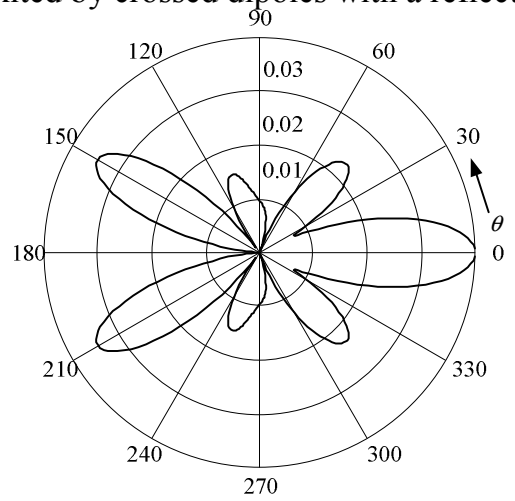

Fig. 2. Radiation pattern for cross-polarisation of Luneburg lens exIted by crossed dipoles with a reflector

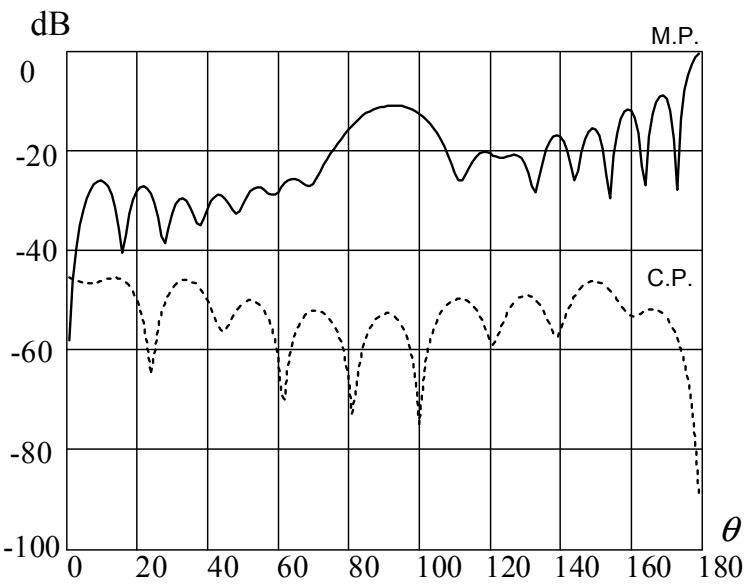

Fig. 3. Radiation pattern of Luneburg lens exited by aperture antenna, $k_{0} a=8 \pi$. Main polarization (solid line), Cross-polarization (dash line)

Radiation patterns of Luneburg lens excited by aperture antenna with circular polarization are shown in Fig.3. Comparison between the main polarization and cross-polarized radiation field level may be simply analyzed.

\section{CONCLUSION}

The universal method based on Green's functions of layered structures for the spherical and cylindrical Luneburg lenses radiation field calculations is suggested.

\section{ACKNOWLEDGEMENT}

This work was funded by the grant of the Russian Science Foundation (project № 14-19-01396).

\section{REFERENCES}

[1] R.K. Luneberg, The Mathematical Theory of Optics. Providence. RT: Brown Univ. Press, 1944.

[2] S. R. Baev, S. M. Gechev, B. N. and P. I. DankovHadjistamov, "Modeling and Simulations of Lüneburg Lens Antennas for Communication Purposes", Proc. TELFOR 2008, $16^{\text {th }}$ Telecommunication Forum, Belgrade, Serbia, pp. 488-491.

[3] T.-T. Chia and W.-Y. Lim, "Design of Low Profile Cylindrical Luneburg Lens Antenna", Proc. EuCAP, Amsterdam, The Netherlands, 2014, pp. 3011-3013.

[4] S. Knyazev, Yu. Kostitsyn and S. Shabunin, Green's Function Formulation for Multilayered Cylindrical Structures and Its Application to Scattering Problems

[5] B. Panchenko, M. Gizatullin, "Synthesis of plane and curved multilayer electromagnetic absorbers". Proc. EuCAP, Edinburgh, Great Britan, 2007, pp. 518-520.

[6] L. Felsen, N. Marcuvitz, Radiation and Scattering of Waves, vol.1. Prentice-Hall, Inc., Englewood Cliffs, New Jersey, 1973. 